\title{
Budget Impact of Dabrafenib and Trametinib in Combination as Adjuvant Treatment of BRAF V600E/K Mutation-Positive Melanoma from a U.S. Commercial Payer Perspective
}

\author{
Daniel Stellato, BS; Margaret E. Gerbasi, PhD; Briana Ndife, MPH; Sameer R. Ghate, PhD; \\ Aaron Moynahan, MA; Dinesh Mishra, MSc; Praveen Gunda, MS; Roy Koruth, MSc; \\ and Thomas E. Delea, MSIA
}

\begin{abstract}
BACKGROUND: Before the approval of dabrafenib and trametinib in combination, there were no approved therapies in the adjuvant setting that target the RAS/RAF/MEK/ERK pathway.

OBJECTIVE: To evaluate the budget impact of dabrafenib and trametinib in combination for adjuvant treatment of patients with BRAF V600 mutationpositive resected Stage IIIA, IIIB, or IIIC melanoma from a U.S. commercial payer perspective using data from the COMBI-AD trial, as well as other sources.

METHODS: The budget impact of dabrafenib and trametinib in combination for patients with BRAF V600E/K mutation-positive, resected Stage IIIA, IIIB, or IIIC melanoma was evaluated from the perspective of a hypothetical population of 1 million members with demographic characteristics consistent with those of a commercially insured U.S. insurance plan (i.e., adults aged less than 65 years) using an economic model developed in Microsoft Excel. The model compared melanoma-related health care costs over a 3-year projection period under 2 scenarios: (1) a reference scenario in which dabrafenib and trametinib are assumed to be unavailable for adjuvant therapy and (2) a new scenario in which the combination is assumed to be available. Treatments potentially displaced by dabrafenib and trametinib were assumed to include observation, high-dose interferon alpha- $2 b$, ipilimumab, and nivolumab. Costs considered in the model include those of adjuvant therapies and treatment of locoregional and distant recurrences. The numbers of patients eligible for treatment with dabrafenib and trametinib were based on data from cancer registries, published sources, and assumptions. Treatment mixes under the reference and new scenarios were based on market research data, clinical expert opinion, and assumptions. Probabilities of recurrence and death were based on data from the COMBI-AD trial and an indirect treatment comparison. Medication costs were based on wholesale acquisition cost prices. Costs of distant recurrence were from a health insurance claims study.
\end{abstract}

RESULTS: In a hypothetical population of 1 million commercially insured members, 48 patients were estimated to become eligible for treatment with dabrafenib and trametinib in combination over the 3-year projection period; in the new scenario, 10 patients were projected to receive such treatment. Cumulative costs of melanoma-related care were estimated to be $\$ 6.3$ million in the reference scenario and $\$ 6.9$ million in the new scenario. The budget impact of dabrafenib and trametinib in combination was an increase of $\$ 549$ thousand overall and 1.5 cents per member per month.

CONCLUSIONS: For a hypothetical U.S. commercial health plan of 1 million members, the budget impact of dabrafenib and trametinib in combination as adjuvant treatment for melanoma is likely to be relatively modest and within the range of published estimates for oncology therapies. These results may assist payers in making coverage decisions regarding the use of adjuvant dabrafenib and trametinib in melanoma.

J Manag Care Spec Pharm. 2019;25(11):1227-35

Copyright $\odot 2019$, Academy of Managed Care Pharmacy. All rights reserved.

\section{What is already known about this subject}

Treatment of metastatic melanoma has been revolutionized in recent years by the introduction of novel targeted and immunotherapies.

The phase 3 COMBI-AD trial demonstrated that adjuvant therapy with dabrafenib and trametinib in combination in patients with completely resected v-Raf murine sarcoma viral oncogene homo$\log$ B (BRAF) mutation-positive Stage IIIA-C melanoma improved relapse-free survival relative to placebo.

\section{What this study adds}

The budget impact of dabrafenib and trametinib in patients with completely resected BRAF mutation-positive Stage IIIA-C melanoma in a U.S. commercial health plan was examined.

Over the 3-year projection, 48 patients would be eligible for and 10 patients initiate therapy with dabrafenib and trametinib, with a budget impact of 1.5 cents per-member per-month.

The budget impact of dabrafenib and trametinib in this setting is within the range of recent estimates for oncology therapies and less than publicly available threshold values.

T reatment of metastatic melanoma has been revolutionized in recent years with the development and introduction of immune checkpoint inhibitors, as well as therapies targeted at the mitogen-activated protein kinase (MAPK) pathway. These treatments have been shown to improve progression-free survival (PFS) and overall survival (OS) in treatment-naive patients with unresectable or metastatic melanoma. ${ }^{1,2}$ Given these favorable results in the metastatic setting, the use of these agents, alone or in combination, has been evaluated in the adjuvant setting in numerous phase 3 trials.

Dabrafenib is a small-molecule targeted inhibitor of $\mathrm{v}$-Raf murine sarcoma viral oncogene homolog B (BRAF). Trametinib is a small-molecule targeted inhibitor of MEK kinase. Because resistance to BRAF inhibitors is associated with reactivation of the MAPK pathway, there is a strong biological rationale for combined treatment with BRAF and MEK inhibitors. COMBI-AD is an ongoing randomized controlled trial of 


\section{TABLE 1 Calculation of Number of Eligible Patients}

\begin{tabular}{|c|c|c|c|c|}
\hline \multirow[b]{3}{*}{ Members } & \multirow[b]{3}{*}{ Estimate } & \multicolumn{2}{|l|}{ Number } & \multirow[b]{2}{*}{ Year 3} \\
\hline & & Year 1 & Year 2 & \\
\hline & & $1,000,000$ & $1,000,000$ & $1,000,000$ \\
\hline \multicolumn{5}{|l|}{ Eligible patients } \\
\hline \multicolumn{5}{|l|}{ Newly diagnosed with Stage III melanoma during projection period } \\
\hline Annual incidence of melanoma per $100 \mathrm{k}^{5}$ & 17 & 171.6 & 171.6 & 171.6 \\
\hline Stage IIIA-C ${ }^{5}$ & $7.6 \%$ & 13.0 & 13.0 & 13.0 \\
\hline Successfully resected 20 & $90.0 \%$ & 11.7 & 11.7 & 11.7 \\
\hline $\mathrm{BRAF}+7-19 \mathrm{a}$ & $40.8 \%$ & 4.8 & 4.8 & 4.8 \\
\hline \multicolumn{5}{|c|}{ Initially diagnosed with Stage I-II melanoma who progress to Stage III during projection period } \\
\hline Previously diagnosed melanoma per 100k (prevalence-incidence) ${ }^{5}$ & 277 & $2,769.6$ & $2,769.6$ & $2,769.6$ \\
\hline Stage I-II ${ }^{6}$ & $90.0 \%$ & $2,491.9$ & $2,491.9$ & $2,491.9$ \\
\hline Progress to Stage IIIA-C annually ${ }^{41}$ & $1.1 \%$ & 26.4 & 26.4 & 26.4 \\
\hline Successfully resected 20 & $90.0 \%$ & 23.7 & 23.7 & 23.7 \\
\hline $\mathrm{BRAF}+7-19 \mathrm{a}$ & $40.8 \%$ & 9.7 & 9.7 & 9.7 \\
\hline \multicolumn{5}{|l|}{ Stage III at model time 0 who experience LR during projection period } \\
\hline Previously diagnosed melanoma per 100k (prevalence-incidence) ${ }^{5}$ & 277 & $2,769.6$ & $2,769.6$ & $2,769.6$ \\
\hline Stage III 6 & $8.4 \%$ & 232.3 & 227.9 & 223.6 \\
\hline Experience recurrence, annually ${ }^{b}$ & $1.7 \%$ & 4.0 & 4.0 & 3.9 \\
\hline Successfully resected 20 & $90.0 \%$ & 3.6 & 3.6 & 3.5 \\
\hline $\mathrm{BRAF}+7-19 \mathrm{a}$ & $40.8 \%$ & 1.5 & 1.5 & 1.4 \\
\hline Total number of eligible patients & & 16.0 & 15.9 & 15.9 \\
\hline \multicolumn{5}{|l|}{$\begin{array}{l}\text { aPart of the total number of eligible patients. } \\
\text { bData on file, Novartis, } 2018 \text {. } \\
\text { BRAF+=BRAF mutation positive; } L R=\text { locoregional recurrence. }\end{array}$} \\
\hline
\end{tabular}

dabrafenib and trametinib in combination versus placebo in Stage IIIA-C patients with BRAF mutation-positive, resected melanoma. Initial results of COMBI-AD demonstrated that dabrafenib and trametinib improved relapse-free survival (RFS) in these patients. ${ }^{3,4} \mathrm{~A}$ favorable hazard ratio was also reported for OS, though the prespecified interim threshold for significance was not reached. ${ }^{3,4}$ Based on the results of COMBI-AD, dabrafenib and trametinib in combination has been approved in the United States as adjuvant treatment of patients with melanoma with BRAF V600E or V600K mutations, as detected by a test approved by the U.S. Food and Drug Administration, and involvement of lymph nodes, following complete resection.

Although COMBI-AD provided evidence sufficient for the regulatory approval of dabrafenib and trametinib in combination as adjuvant treatment of melanoma, health care payers require information on the budget impact associated with introducing new pharmaceutical products into their formularies. For this purpose, we evaluated the budget impact of introducing dabrafenib and trametinib in combination as adjuvant treatment of patients with melanoma to a hypothetical U.S. health plan of 1 million adults aged less than 65 years.

\section{Methods}

\section{Overview}

The objective of this study was to evaluate the budget impact of dabrafenib and trametinib in combination as adjuvant treatment for melanoma from a U.S. commercial health plan perspective (i.e., adults aged less than 65 years) based on the COMBI-AD trial and other sources. The eligible population was defined as patients with resected Stage IIIA-C melanoma who are BRAF mutation-positive and who have not received prior systemic therapy. The budget impact of dabrafenib plus trametinib was calculated using an economic model developed in Excel (Microsoft, Redmond, WA) and was defined as the difference in expected costs of treatment of melanoma in the eligible population under 2 different scenarios: a reference scenario, in which it is assumed that combination dabrafenib plus trametinib is not available on the health plan formulary, and a new scenario, in which it is assumed that dabrafenib plus trametinib is available. Other adjuvant treatments considered in the model, the use of which may be displaced by dabrafenib and trametinib, included observation, high-dose interferon alfa-2b, ipilimumab, and nivolumab. A 3-year modeling time horizon was used. 
Budget Impact of Dabrafenib and Trametinib in Combination as Adjuvant Treatment of BRAF V600E/K Mutation-Positive Melanoma from a U.S. Commercial Payer Perspective

TABLE 2 Estimated Treatment Mix Under Reference and New Scenarios for All Eligible Patients

\begin{tabular}{|c|c|c|c|c|c|c|}
\hline \multirow[b]{2}{*}{ Therapy } & \multicolumn{3}{|c|}{ Reference Scenario, \% } & \multicolumn{3}{|c|}{ New Scenario, \% } \\
\hline & Year 1 & Year 2 & Year 3 & Year 1 & Year 2 & Year 3 \\
\hline Dabrafenib and trametinib & 0.0 & 0.0 & 0.0 & 10.0 & 20.0 & 30.0 \\
\hline Observation & 72.7 & 46.3 & 20.0 & 65.4 & 37.1 & 20.0 \\
\hline High-dose interferon & 0.5 & 0.5 & 0.5 & 0.5 & 0.4 & 0.4 \\
\hline Ipilimumab & 0.5 & 0.5 & 0.5 & 0.5 & 0.4 & 0.4 \\
\hline Nivolumab & 26.3 & 52.7 & 79.0 & 23.7 & 42.1 & 49.3 \\
\hline
\end{tabular}

\section{Model Description}

The model consists of 3 modules: (1) the population module, which calculates the number of eligible patients who initiate various adjuvant treatments for melanoma in each year under the reference and new scenarios; (2) the costs module, which generates estimates of melanoma-related health care costs by time since initiation of therapy for different adjuvant therapies; and (3) the results module, which combines estimates from the population and costs modules to generate estimates of budget impact. The cost module uses a nonhomogeneous semi-Markov cohort model with a 6-month cycle-duration and 4 health states: (1) RFS, (2) locoregional recurrence (LR), (3) distant recurrence (DR), and (4) death. Patients initiating treatment are assumed to enter the model in the RFS state, from which they may transition to the LR, DR, or death states. Patients in the LR state may transit to the DR or death states, while patients in the DR state may transit to the death state. Probabilities and costs are conditioned on health state, time elapsed since entry into health state, and time since initiation of adjuvant therapy.

\section{Eligible Population}

The population of patients who are eligible to receive adjuvant treatment with dabrafenib and trametinib in each year of the projection is assumed to consist of 3 groups: (1) patients with newly diagnosed BRAF mutation-positive, Stage III resectable melanoma; (2) patients with previously diagnosed BRAF mutation-positive, Stage I or II melanoma who progress to Stage III resectable melanoma during the year; and (3) patients with previously diagnosed BRAF mutation-positive, Stage III melanoma who experience an LR during the year. For group 3, only those patients who have Stage III melanoma at model time zero are considered, as the costs module captures the costs of treatment for eligible patients who experience an LR. Calculations of the number of patients who would be eligible for treatment with dabrafenib and trametinib in each year are displayed in Table 1. Estimates of the annual incidence and the stage distribution of newly diagnosed cases were from the Surveillance, Epidemiology, and End Results (SEER) program of the National Cancer Institute. ${ }^{5}$ Estimates of the prevalence and stage distribution of prevalent melanoma were from a study by Lin et al. (2011) that used a multicohort natural disease history model based on data from SEER and other sources. ${ }^{6}$ Estimates of the percentage of melanoma patients with BRAF mutation were based on a meta-analysis of studies identified by a targeted review of the literature. ${ }^{7-19}$ Estimates of the proportion of Stage III cases that are successfully resected (90\%) were based on clinical expert opinion and data from SEER. ${ }^{20}$ It was conservatively assumed that all eligible patients would be treatment naive.

BRAF mutation testing was assumed to potentially vary by year of the projection and to differ for the reference and new scenarios. In the reference scenario, it was assumed that $10 \%$ of patients in the eligible population would undergo BRAF testing each year based on results of a study of health insurance claims data. ${ }^{21}$ It was assumed that, as a result of the introduction of dabrafenib plus trametinib as adjuvant therapy, testing rates in the new scenario would increase linearly from $20 \%$ in year 1 to $60 \%$ in year 3. All eligible patients who were tested were assumed to test positive (the test was assumed to be $100 \%$ sensitive).

\section{Treatment Mix}

Estimates of the proportion of patients receiving each treatment by year under the reference and new scenarios (i.e., the "treatment mix") were based on clinical expert opinion supplemented with unpublished market research data (data on file, Novartis, 2018; Table 2). For the reference scenario, it was assumed that the treatment mix would be the same for tested and untested patients (i.e., those with known and unknown BRAF mutation). The market share of observation was assumed to not be less than 20\% in any year of the projection, both interferon alpha-2b and ipilimumab were assumed to be used in $0.5 \%$ of patients each, and the market share for nivolumab was assumed to increase linearly from year 1 to year 3, reaching a maximum of $79 \%$ (i.e., the complement of market shares for other therapies). Therefore, use of nivolumab was assumed to increase from 26\% in year 1 to $79 \%$ in year 3 and would displace use of observation. The proportion of patients who would receive observation alone was assumed to decline from $73 \%$ in year 1 to $20 \%$ in year 3, whereas use of high-dose interferon alpha-2b and ipilimumab were assumed to be constant at $0.5 \%$ each from 


\begin{tabular}{|c|c|c|}
\hline Cost Component & Value & Source \\
\hline BRAF mutation test (cost per test), $\$$ & 180 & 43 \\
\hline BRAF mutation test invalid rate, $\%$ & 9 & 44 \\
\hline \multicolumn{3}{|l|}{ Medications (\$ per mg) } \\
\hline Dabrafenib & 1 & 27 \\
\hline Trametinib & 175 & 27 \\
\hline High-dose interferon & 28 & 27 \\
\hline Ipilimumab & 0 & 27 \\
\hline Nivolumab & 25 & 27 \\
\hline \multicolumn{3}{|l|}{ Administration of medications, $\$$} \\
\hline IV therapy, 1-hour & 139.61 & 44 \\
\hline IV therapy, 1.5-hour & 168.32 & 44 \\
\hline Subcutaneous therapy & 25.84 & 44 \\
\hline Drug dispensing fee & 11.77 & 45 \\
\hline Acute treatment of LR & 5,520 & 21 \\
\hline \multicolumn{3}{|l|}{ Other (\$ per month), adjuvant setting } \\
\hline Years $1-2$ & 1,012 & 21 \\
\hline Years 3-5 & 506 & 21 \\
\hline \multicolumn{3}{|l|}{ DR, by time (months) since $\mathrm{DR}, \$$} \\
\hline $1-6$ & 17,450 & 21 \\
\hline $7-12$ & 7,826 & 21 \\
\hline $12+$ & 5,603 & 21 \\
\hline Terminal care & 16,783 & 46 \\
\hline
\end{tabular}

$D R=$ distal recurrence; $I V=$ intravenous; $L R=$ locoregional recurrence;

year 1 to year 3. Under the new scenario, it was assumed 50\% of tested patients would receive dabrafenib plus trametinib and, therefore, that $10 \%, 20 \%$, and $30 \%$ of all eligible patients (i.e., those with a BRAF mutation, known or unknown) would receive dabrafenib plus trametinib in year 1 , year 2 , and year 3 , respectively. It was assumed that, among tested patients, dabrafenib and trametinib would displace observation and nivolumab proportionately in years 1 and 2, but that in year 3, the displacement would be from active therapies only (mostly nivolumab, with a small amount of displacement of high-dose interferon alpha-2b and ipilimumab). Alternative assumptions regarding the displacement of other therapies were explored in sensitivity analyses. For eligible patients who did not undergo BRAF mutation testing, the treatment mix was assumed to be the same as if dabrafenib plus trametinib was not available (i.e., the same as for the reference scenario).

\section{Transition Probabilities}

Transition probabilities for the Markov model used to estimate the costs of melanoma care for patients initiating adjuvant therapy were estimated using data from the COMBI-AD trial and other studies. These included the probabilities of RFS events (LR, DR, or death), subsequent recurrence events for patient with LR (DR or death), and death for patients with DR. Probabilities of RFS events for dabrafenib plus trametinib and observation were based on parametric survival distributions fit to individual patient-level data from COMBI-AD using Flexsurv, an $\mathrm{R}$ package for fully parametric modelling of survival data (R Foundation for Statistical Computing, Vienna, Austria). ${ }^{22}$ A variety of parametric distributions were considered, including exponential, Weibull, log-logistic, lognormal, Gompertz, gamma, generalized F, and restricted cubic spline distributions. In these analyses, patients with second primary malignancy without concurrent LR or DR events were censored. For RFS, a log-logistic unrestricted cure distribution was selected for the base case based on assessment of fit statistics and visual fit to Kaplan-Meier survival plots. RFS for other comparators was estimated by applying to the estimated RFS distribution for observation from COMBI-AD estimated hazard ratios (HRs) for RFS for each of the comparators vs. observation from an indirect treatment comparison (ITC) of COMBI-AD, ${ }^{3}$ the Checkmate 238 trial of nivolumab versus ipilimumab,,$^{19}$ the European Organisation for Research and Treatment of Cancer 18071 trial of ipilimumab versus placebo, ${ }^{23}$ and the Eastern Cooperative Oncology Group 1684 and E1690 trials of high-dose interferon alpha-2b versus observation. ${ }^{24,25}$ For Checkmate 238, the HR for RFS was based on that reported for patients with BRAF mutation (HRs for RFS by BRAF mutation were not available for trials of ipilimumab or interferon alpha-2b). Based on these trials, the HRs for RFS versus observation were estimated to be 0.76 (95\% [confidence interval] $\mathrm{CI}=0.64-0.89$ ) for ipilimumab, 0.77 (95\% CI $=0.62-0.97)$ for high-dose interferon alpha-2b, and 0.55 (95\% CI $=0.38-0.79)$ for nivolumab.

Because patients in COMBI-AD were not followed for subsequent DR after LR, it was not feasible to estimate probabilities of DR or death after LR using data from COMBI-AD. These probabilities were therefore estimated by applying to the probability of RFS events an estimate of the increased risk of recurrence following LR reported in a surveillance study by Salama et al. (2013). ${ }^{26}$ The probability of death after DR was assumed to be the same regardless of metastatic treatment received and was based on a Gompertz distribution fit to individual patient data from COMBI-AD. The probability distribution of RFS events by type were assumed to be dependent on adjuvant treatment and estimated based on data from COMBI-AD. For patients receiving initial adjuvant therapy other than dabrafenib plus trametinib or observation, the distribution of RFS events by type was assumed to be the same as that for observation.

\section{Costs}

Costs of BRAF mutation testing were based on published estimates and were applied to all patients tested in the adjuvant setting and to patients with DR who had not been tested previously. Costs of testing were adjusted to reflect the percentage of tests anticipated to yield an invalid test and therefore require retesting. Costs of medication were based on 2017 wholesale acquisition cost prices. ${ }^{27}$ For drugs for which 
multiple formulations were available, the formulation with the lowest cost per milligram was used. Ipilimumab as adjuvant treatment was assumed to be available at no cost based on the Yervoy Adjuvant Patient Program for Melanoma. ${ }^{28}$ Dosage schedules were from pivotal studies and approved prescribing information. ${ }^{19,23,24,29,30}$ Relative dose intensities (RDI) for dabrafenib (0.838) and trametinib (0.905) were calculated as the mean daily dose divided by the intended daily dose from COMBI-AD (data on file, Novartis, 2017). The RDI for high-dose interferon alfa-2b was calculated based on mean daily doses reported for the E1684 and E1690 trials (induction: 0.9125; maintenance 0.815). ${ }^{24,25}$ In the absence of published data on dose intensity for the checkpoint inhibitors, RDIs for ipilimumab and nivolumab were assumed to be 1.00. Monthly costs of follow-up and monitoring in the adjuvant setting, one-off costs of acute treatment of LR, and costs of treating DR (including medication costs) by time, since DR were from a retrospective study of a large health insurance claims database ${ }^{21}$ The probabilities of grades 3-5 adverse events (AE) with incidence $\geq 5 \%$ for any of the treatment strategies were based on pooled estimates obtained from phase 3 clinical trials conducted in the adjuvant setting (Appendix A, available in online article). Costs of AEs were based on published studies (Appendix B, available in online article). All costs were adjusted to 2017 U.S. dollars using the consumer price index for medical care. Cost inputs used in the model are shown in Table 3.

\section{Analyses}

Base case analyses were conducted for a hypothetical commercially insured population of 1 million U.S. adults aged less than 65 years. Costs for the reference and new scenarios were reported by health state, category of service, and year of the projection. Budget impact in each year was calculated as the difference in total costs between scenarios and was calculated overall and on a per member per month (PMPM) basis. Sensitivity analyses were conducted to assess the effects on budget impact of varying model parameters. Scenario analyses were conducted in which only costs of adjuvant treatment were considered and in which only medication costs were considered. A scenario analysis also was conducted in which dabrafenib plus trametinib first displaces the use of observation before active therapies, with displacement of active therapies proportional to their use. To assess the impact of our assumption that the RDI for nivolumab and ipilimumab are 100\%, budget impact was calculated for a scenario assuming an RDI of 0.75 for these therapies. An exploratory scenario was also conducted for a hypothetical U.S. insurance plan of 1 million members aged $\geq 65$ years and with costs of DR estimated using data for Medicare patients. ${ }^{21}$

\section{Results}

\section{Base Case}

For a hypothetical population of 1 million persons with demographic characteristics similar to the commercially insured U.S. adult population, it was estimated that 4.8 patients would be newly diagnosed with Stage IIIA-C, BRAF mutation-positive melanoma and successfully resected in each year of the projection. Another 9.7 patients with Stage I-II, BRAF mutation-positive melanoma were estimated to experience disease relapse (i.e., progress to Stage III) and subsequently undergo successful resection annually. In addition, approximately 1.5 prevalent patients (i.e., previously diagnosed) with Stage III, BRAF mutation-positive melanoma were estimated to experience LR and be successfully resected in each year. On balance, it was estimated that 16 patients with resected, BRAF mutation-positive, Stage III melanoma would become eligible for adjuvant treatment with dabrafenib and trametinib in each year of the projection, or a total of 48 patients over 3 years.

In the reference scenario, it was projected that 25 of these patients would receive nivolumab, $<1$ each would receive interferon alpha-2b and ipilimumab, and 22 would receive observation over the 3 -year projection period. In the new scenario, it was projected that 19 eligible patients would undergo BRAF mutation testing, while 29 would not undergo testing. Assuming 50\% of patients known to be BRAF mutationpositive would initiate adjuvant therapy with dabrafenib and trametinib in combination, it was estimated that 10 patients would receive dabrafenib and trametinib in combination, 18 would receive nivolumab, $<1$ each would receive interferon alpha-2b and ipilimumab, and 20 would receive observation.

With respect to clinical outcomes, it was estimated that 7, 12, and 2 patients would experience LR, DR, and death, respectively, over the projection period under the reference scenario compared with 6,11 , and 2 such events in the new scenario. The introduction of dabrafenib and trametinib in combination as adjuvant therapy was thus projected to result in 1 fewer LR and DR each over the 3-year projection period.

The cost per course (medication and administration costs) of adjuvant therapy was estimated to be $\$ 170,728$ for dabrafenib and trametinib, $\$ 140,628$ for nivolumab, $\$ 1,280$ for ipilimumab (administration costs only), and $\$ 75,135$ for interferon alpha2b. For the hypothetical population of 1 million commercially insured members, total melanoma-related health care costs over the 3-year projection period were estimated to be $\$ 6.3$ million in the reference scenario and $\$ 6.9$ million in the new scenario (Table 4). The budget impact of dabrafenib and trametinib was therefore estimated to be an increase of $\$ 549,000$ over 3 years $(\$ 165,841, \$ 253,376$, and $\$ 129,389$ in years 1 , 2 , and 3 , respectively). The lower budget impact in year 3 versus year 2 reflects projected increasing cost savings from LRs and DRs prevented, as well as increasing displacement 
Budget Impact of Dabrafenib and Trametinib in Combination as Adjuvant Treatment of BRAF V600E/K Mutation-Positive Melanoma from a U.S. Commercial Payer Perspective

TABLE 4 Base Case Results: Costs by Scenario, Year, Disease Stage, and Cost Category

\begin{tabular}{|c|c|c|c|c|c|c|c|c|c|c|c|c|}
\hline \multirow[b]{2}{*}{ Costs, $\$$} & \multicolumn{4}{|c|}{ Reference Scenario } & \multicolumn{4}{|c|}{ New Scenario } & \multicolumn{4}{|c|}{ Incremental } \\
\hline & Year 1 & Year 2 & Year 3 & Total & Year 1 & Year 2 & Year 3 & Total & Year 1 & Year 2 & Year 3 & Total \\
\hline \multicolumn{13}{|l|}{ Relapse-free survival } \\
\hline $\begin{array}{l}\text { Follow-up and } \\
\text { monitoring }\end{array}$ & 132,842 & 253,223 & 346,961 & 733,027 & 133,931 & 260,047 & 360,655 & 754,633 & 1,088 & 6,824 & 13,694 & 21,607 \\
\hline Medications ${ }^{\mathrm{a}}$ & 468,600 & $1,058,741$ & $1,645,843$ & $3,173,184$ & 638,666 & $1,348,398$ & $1,831,643$ & $3,818,706$ & 170,065 & 289,657 & 185,800 & 645,522 \\
\hline Adverse events & 5,323 & 5,296 & 5,270 & 15,889 & 7,747 & 10,138 & 12,529 & 30,414 & 2,423 & 4,842 & 7,259 & 14,525 \\
\hline Total & 606,766 & $1,317,260$ & $1,998,074$ & $3,922,100$ & 780,343 & $1,618,584$ & $2,204,827$ & $4,603,754$ & 173,577 & 301,323 & 206,753 & 681,654 \\
\hline \multicolumn{13}{|c|}{ Locoregional recurrence } \\
\hline $\begin{array}{l}\text { Follow-up and } \\
\text { monitoring }\end{array}$ & 5,514 & 27,299 & 41,461 & 74,273 & 5,014 & 25,061 & 37,974 & 68,049 & -500 & $-2,238$ & $-3,487$ & $-6,224$ \\
\hline Medications $^{\mathrm{a}}$ & 17,135 & 146,000 & 215,050 & 378,185 & 19,736 & 155,330 & 232,629 & 407,694 & 2,601 & 9,329 & 17,578 & 29,509 \\
\hline Adverse events & 486 & 2,671 & 5,958 & 9,114 & 495 & 2,429 & 4,290 & 7,214 & 9 & -241 & $-1,667$ & $-1,900$ \\
\hline $\begin{array}{l}\text { Acute treatment of } \\
\text { recurrence }\end{array}$ & 5,012 & 16,435 & 17,636 & 39,083 & 4,558 & 14,921 & 15,742 & 35,221 & -454 & $-1,514$ & $-1,894$ & $-3,862$ \\
\hline Total & 28,147 & 192,405 & 280,104 & 500,656 & 29,803 & 197,741 & 290,634 & 518,179 & 1,656 & 5,337 & 10,530 & 17,523 \\
\hline Distant recurrence & 118,172 & 676,026 & $1,067,333$ & $1,861,532$ & 108,058 & 621,725 & 979,030 & $1,708,813$ & $-10,114$ & $-54,301$ & $-88,304$ & $-152,719$ \\
\hline BRAF testing & 964 & 1,639 & 1,841 & 4,444 & 1,693 & 3,592 & 5,017 & 10,302 & 728 & 1,954 & 3,176 & 5,858 \\
\hline Terminal care & 142 & 12,728 & 35,666 & 48,536 & 136 & 11,792 & 32,899 & 44,826 & -6 & -936 & $-2,767$ & $-3,710$ \\
\hline Total & 754,192 & $2,200,058$ & $3,383,018$ & $6,337,267$ & 920,033 & $2,453,434$ & $3,512,406$ & $6,885,874$ & 165,841 & 253,376 & 129,389 & 548,606 \\
\hline $\begin{array}{l}\text { Per member per } \\
\text { month }\end{array}$ & 0.0628 & 0.1833 & 0.2819 & 0.176 & 0.0767 & 0.2045 & 0.2927 & 0.1913 & 0.0138 & 0.0211 & 0.0108 & 0.0152 \\
\hline
\end{tabular}

Note: Due to rounding, there may be small differences between the sum of individual cost components and the total costs.

ancludes administration and dispensing costs.

of active therapies (in particular, nivolumab) over time. Over the 3-year projection period, PMPM costs were estimated to increase by 1.5 cents from 17.6 cents in the reference scenario to 19.1 cents in the new scenario.

\section{Sensitivity Analyses}

Results of sensitivity analyses on the cumulative budget impact over the 3-year projection period are presented as a tornado diagram in Figure 1. Budget impact was sensitive to estimates of the utilization of dabrafenib and trametinib, the cost of dabrafenib and trametinib, the rates of BRAF mutation testing, the cost of DR, and the cure fraction for RFS for dabrafenib and trametinib. Scenario analyses indicate that consideration of adjuvant costs only would result in an average increase in PMPM costs of 1.4 cents over the 3-year projection period. Consideration of medication costs only would result in an average increase in PMPM costs of 1.5 cents. Assuming that dabrafenib plus trametinib first displaces the use of observation before active therapies, with displacement of active therapies proportional to their use, the budget impact of introducing dabrafenib and trametinib is 2.7 cents on a PMPM basis over the 3-year projection period. Assuming that the RDI for nivolumab and ipilimumab is 0.75 , the budget impact of dabrafenib and trametinib is 2.1 cents PMPM over the 3 -year projection period. In a scenario for a hypothetical insurance plan of 1 million members aged $\geq 65$ years and with costs of DR estimated using data for Medicare patients, it was estimated that there would be 51 eligible patients in each year (153 over the entire projection) and that the introduction of dabrafenib and trametinib as adjuvant therapy would increase PMPM costs by 4.9 cents. The relatively larger impact in the Medicare population is due to the higher incidence and prevalence of melanoma among elderly patients.

\section{Discussion}

This study evaluated the budget impact of dabrafenib and trametinib in combination as adjuvant treatment of patients with BRAF V600 mutation-positive, Stage IIIA-C resected melanoma. For a hypothetical commercially insured plan of 1 million members, it was estimated that 48 patients would become eligible for treatment with dabrafenib and trametinib in combination over the 3-year projection period. Assuming that use of dabrafenib plus trametinib among eligible patients increases from $10 \%$ in year 1 to $30 \%$ in year 3, 10 patients would receive dabrafenib and trametinib over the projection period, and melanoma-related health care costs in the plan would increase by $\$ 549,000$ (1.5 cents PMPM) relative to the reference scenario in which dabrafenib and trametinib is assumed to be unavailable. Deterministic sensitivity analyses indicate that budget impact is sensitive to estimates of the relative dose intensities, utilization of dabrafenib and trametinib, the cost of dabrafenib and trametinib, the rates of BRAF mutation testing, the cost of DR, and the cure fraction for RFS for dabrafenib and trametinib. Budget impact was greater for a hypothetical population 
Budget Impact of Dabrafenib and Trametinib in Combination as Adjuvant Treatment of BRAF V600E/K Mutation-Positive Melanoma from a U.S. Commercial Payer Perspective

\section{FIGURE 1 Tornado Diagram of Incremental PMPM Costs}

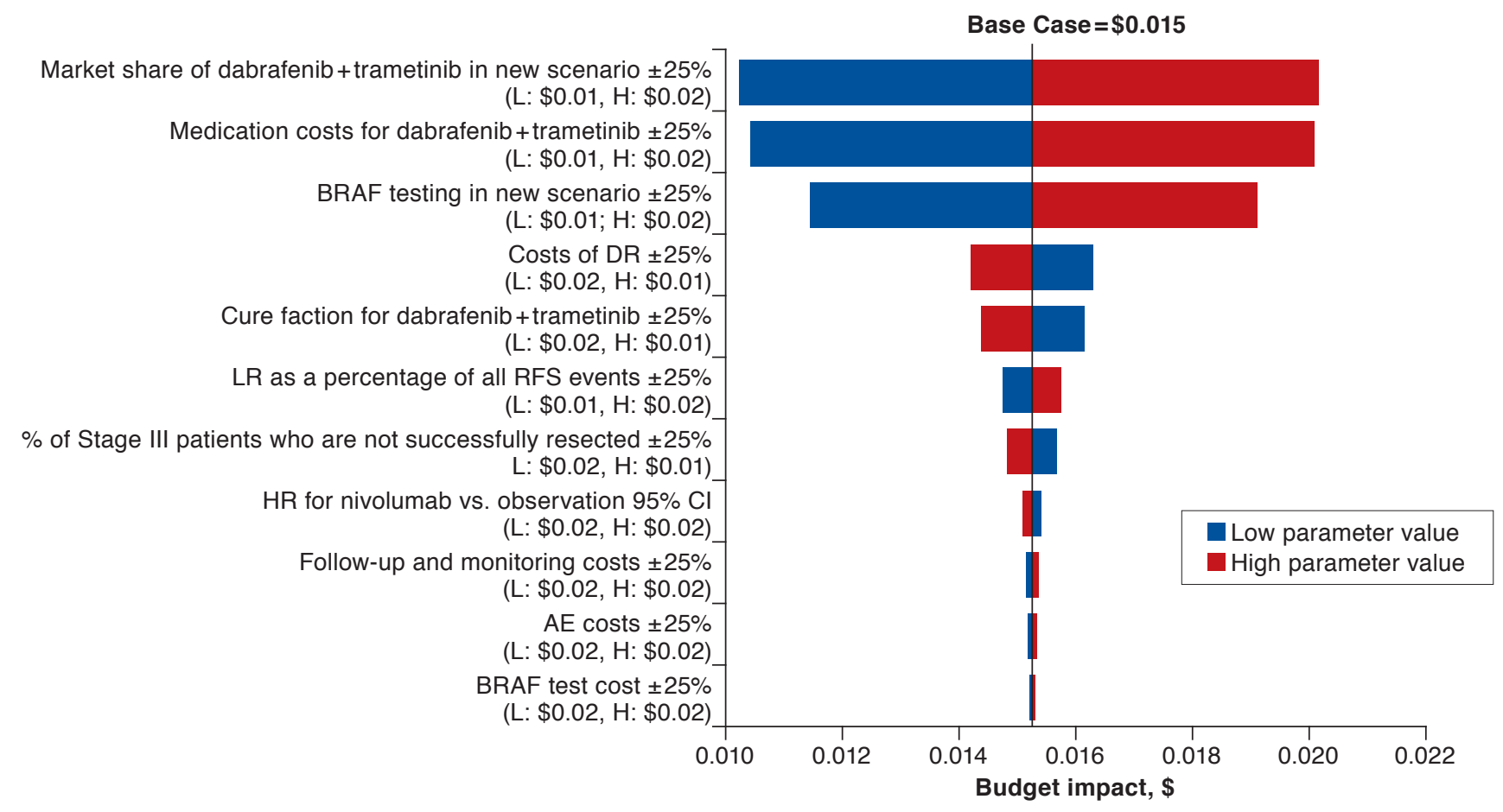

$A E=$ adverse event $C I=$ confidence interval; $D R=$ distant recurrent $H=$ high; $H R=$ hazard ratio; $L=$ low; $L R=$ locoregional recurrence; $P M P M=$ per member per month; RFS = relapse-free survival.

of Medicare patients (4.9 cents PMPM), due to the higher incidence and prevalence of melanoma among elderly patients.

There are no well-established thresholds for budget impact in the United States. Based on a nonsystematic review of recent budget impact analyses of cancer therapies, PMPMs for such therapies range from a savings of $\$ 0.007$ PMPM for cabazitaxel in hormone refractory prostate cancer to $\$ 0.528$ PMPM for cabazitaxel as a second-line treatment for castration-resistant prostate cancer. ${ }^{31-38}$ The Institute for Clinical and Economic Review (ICER) uses an annual budget impact threshold for prescription drug therapies of $\$ 991$ million, ${ }^{39}$ corresponding to PMPM of approximately 25 cents for the entire U.S. population of 325 million persons. It should be noted that the examples cited above represent treatments that have been available for a number of years and may not be representative of novel targeted and immunotherapies. Nevertheless, the PMPM for adjuvant dabrafenib and trametinib (1.5 cents PMPM) is within the range of these examples and is well below the implied PMPM threshold used by ICER. ${ }^{39}$

Based on a targeted review of the published literature, we were unable to identify any other published studies evaluating the budget impact of dabrafenib and trametinib as adjuvant therapy for Stage III melanoma. Stav et al. (2018) reported an estimated annual cost of adjuvant treatment with nivolumab of approximately $\$ 1.15$ billion for the entire target population in the United States assuming full uptake. ${ }^{40}$ Our analysis focused only on commercially insured patients, so it is not possible to directly compare our results with those reported by Stav et al.

\section{Limitations}

Limitations of this analysis should be noted. There is considerable uncertainty around several of the estimates used in the model. The percentage of Stage III patients with BRAF V600E/K was estimated from a targeted review of the published literature in which there was considerable variability. Estimates of the rate of BRAF testing and the treatment mix in the adjuvant setting were based largely on expert opinion and assumption and cannot be directly validated. RFS for active therapies other than dabrafenib and trametinib was estimated by applying an HR versus observation based on an ITC, under the assumption of proportional hazards. This assumption may not be valid across all trials contributing to the ITC. Estimates of survival following DR were based on data from COMBI-AD, and estimates of costs following DR were based on claims data 
from 2007 to 2017; these data sources may not represent current typical clinical practice in the United States.

Costs of medication were based on 2017 prices, which may not reflect current price levels at the time of publication of this study. The model used a 6-month cycle, which may have introduced some imprecision into the model estimates. Due to lack of data, dose intensity for pembrolizumab was assumed to be $100 \%$. To the extent that the actual dose intensity is less than $100 \%$, this would lead to underestimation of budget impact. Because probabilistic sensitivity analyses were not conducted, we were unable to provide estimates of the precision of our results (e.g., 95\% CIs).

Finally, this model does not consider the use of pembrolizumab in the adjuvant setting, although the manufacturer had submitted a supplemental biologics license application for its use as adjuvant therapy at the time of the preparation of this study.

\section{Conclusions}

Despite limitations, the results from this study are important, as they suggest that, for U.S. commercial health plans, the budget impact of dabrafenib and trametinib in combination as adjuvant treatment for melanoma is likely to be relatively modest and within the range of published estimates for oncology therapies. These results may assist payers in making coverage decisions regarding the use of adjuvant dabrafenib and trametinib in melanoma.

\section{Authors}

DANIEL STELLATO, BS; MARGARET E. GERBASI, PhD; AARON MOYNAHAN, MA; and THOMAS E. DELEA, MSIA, Policy Analysis Inc. (PAI), Brookline, Massachusetts. BRIANA NDIFE, MPH; SAMEER R. GHATE, PhD; and ROY KORUTH, MSc, Novartis Pharmaceuticals, East Hanover, New Jersey. DINESH MISHRA, MSc, and PRAVEEN GUNDA, MS, Novartis Pharmaceuticals, Hyderabad, Telangana, India.

AUTHOR CORRESPONDENCE: Thomas E. Delea, MSIA, Policy Analysis Inc., 4 Davis Ct., Brookline, MA 02445. Tel.: 617.232.4400; E-mail:tdelea@pai2.com.

\section{REFERENCES}

1. Eggermont AM, Maio M, Robert C. Immune checkpoint inhibitors in melanoma provide the cornerstones for curative therapies. Semin Oncol. 2015;42(3):429-35.

2. Niezgoda A, Niezgoda P, Czajkowski R. Novel approaches to treatment of advanced melanoma: a review on targeted therapy and immunotherapy. Biomed Res Int. 2015;2015:851387.

3. Long GV, Hauschild A, Santinami M, et al. Adjuvant dabrafenib plus trametinib in stage III BRAF-mutated melanoma. N Engl J Med. 2017;377(19):1813-23.

4. Hauschild A, Santinami M, Long GV, et al. COMBI-AD: adjuvant dabrafenib plus trametinib for resected stage III BRAF V600-mutant melanoma. Ann Oncol. 2017;28(Suppl 5):v605-49.

5. Tarhini A, Ghate S, Ionescu-Ittu R, et al. Stage III melanoma incidence and impact of transitioning to the 8th AJCC staging system: a U.S. population-based study. Future Oncol. 2019;15(4):359-70.

6. Lin AY, Wang PF, Kolker J. 8584 Multicohort model of prevalence estimation of advanced malignant melanoma in the United States: an increasing public health concern [abstract]. J Clin Oncol. 2011;29(15 Suppl):8584.

7. Akman T, Oztop I, Baskin Y, et al. The role of BRAF mutation in patients with high-risk malignant melanoma treated with high-dose adjuvant interferon therapy. Med Oncol. 2015;32(1):440.

8. Barbour AP, Tang YH, Armour N, et al. BRAF mutation status is an independent prognostic factor for resected stage IIIB and IIIC melanoma: implications for melanoma staging and adjuvant therapy. Eur J Cancer. 2014:50(15):2668-76.

9. Boursault L, Haddad V, Vergier B, et al. Tumor homogeneity between primary and metastatic sites for BRAF status in metastatic melanoma determined by immunohistochemical and molecular testing. PLoS One. 2013;8(8):e70826

10. Heppt MV, Siepmann T, Engel J, et al. Prognostic significance of BRAF and NRAS mutations in melanoma: a German study from routine care. BMC Cancer. 2017;17(1):536.

11. Johansson CC, Egyhazi S, Masucci G, et al. Prognostic significance of tumor iNOS and COX-2 in stage III malignant cutaneous melanoma. Cancer Immunol Immunother. 2009;58(7):1085-94.

12. Knol AC, Pandolfino MC, Vallee A, et al. Comparative analysis of BRAF, NRAS and c-KIT mutation status between tumor tissues and autologous tumor cell-lines of stage III/IV melanoma. Exp Dermatol. 2015;24(1):70-73.

13. Mann GJ, Pupo GM, Campain AE, et al. BRAF mutation, NRAS mutation, and the absence of an immune-related expressed gene profile predict poor outcome in patients with stage III melanoma. J Invest Dermatol. 2013;133(2):509-17.

14. Moreau S, Saiag P, Aegerter P, et al. Prognostic value of BRAF V600 mutations in melanoma patients after resection of metastatic lymph nodes. Ann Surg Oncol. 2012;19(13):4314-21.

15. Picard M, Pham Dang N, D'Incan M, et al. Is BRAF a prognostic factor in stage III skin melanoma? A retrospective study of 72 patients after positive sentinel lymph node dissection. Br J Dermatol. 2014;171(1):108-14.

16. Rutkowski P, Gos A, Jurkowska M, et al. Molecular alterations in clinical stage III cutaneous melanoma: correlation with clinicopathological features and patient outcome. Oncol Lett. 2014;8(1):47-54.

17. Shinozaki M, Fujimoto A, Morton DL, Hoon DS. Incidence of BRAF oncogene mutation and clinical relevance for primary cutaneous melanomas. Clin Cancer Res. 2004;10(5):1753-57.

18. Thomas NE, Edmiston SN, Alexander A, et al. Association between NRAS and BRAF mutationalstatus and melanoma-specific survival among patients with higher-risk primary melanoma. JAMA Oncol. 2015;1(3):359-68.

Funding for this research was provided to Policy Analysis Inc. (PAI) by Novartis Pharmaceuticals. Stellato, Moynahan, and Delea are employed by PAI. Ndife, Koruth, Mishra, and Gunda are employed by Novartis. Ghate was employed by Novartis at the time of this study and is shareholder in Novartis, Provectus Biopharmaceuticals, and Mannkind Corporation. Gerbasi was employed by PAI at the time of this study and is currently an employee, and stockholder, of Sage Therapeutics. Delea reports grant funding from Merck and research funding from Amgen, Novartis, Sanofi, Seattle Genetics, Takeda, Jazz, EMD Serono, and 21st Century Oncology, unrelated to this work. 


\section{Budget Impact of Dabrafenib and Trametinib in Combination as Adjuvant Treatment of BRAF V600E/K Mutation-Positive Melanoma from a U.S. Commercial Payer Perspective}

19. Weber J, Mandala M, Del Vecchio M, et al. Adjuvant nivolumab versus ipilimumab in resected stage III or IV melanoma. N Engl J Med. 2017; 377(19):1824-35

20. Surveillance, Epidemiology, and End Results Program. SEER*Stat Database: Incidence-SEER 18 Regs Research Data + Hurricane Katrina Impacted Louisiana Cases, Nov 2018 Sub (2000-2016) < Katrina/Rita Population Adjustment >-Linked To County Attributes-Total U.S., 19692017 Counties. 2018. Available at: https://seer.cancer.gov/data-software/ documentation/seerstat/nov2018/. Accessed October 22, 2019.

21. Tarhini A, Ghate SR, Ionescu-Ittu R, et al. Postsurgical treatment landscape and economic burden of locoregional and distant recurrence in patients with operable nonmetastatic melanoma. Melanoma Res. 2018;28(6):618-28.

22. Jackson C. Flexible parametric survival and multi-state models. May 11, 2016. Available at: https://cran.r-project.org/web/packages/flexsurv/flexsurv. pdf. Accessed September 24, 2019.

23. Eggermont AM, Chiarion-Sileni V, Grob JJ, et al. Prolonged survival in stage III melanoma with ipilimumab adjuvant therapy. N Engl J Med. 2016;375(19):1845-55

24. Kirkwood JM, Strawderman MH, Ernstoff MS, Smith TJ, Borden EC, Blum RH. Interferon alfa-2b adjuvant therapy of high-risk resected cutaneous melanoma: the Eastern Cooperative Oncology Group Trial EST 1684. J Clin Oncol. 1996;14(1):7-17.

25. Kirkwood JM, Ibrahim JG, Sondak VK, et al. High- and low-dose interferon alfa-2b in high-risk melanoma: first analysis of intergroup trial E1690/ S9111/C9190. J Clin Oncol. 2000;18(12):2444-58

26. Salama AK, de Rosa N, Scheri RP, et al. Hazard-rate analysis and patterns of recurrence in early stage melanoma: moving towards a rationally designed surveillance strategy. PLoS One. 2013;8(3):e57665.

27. IBM Watson Health. IBM Micromedex RED BOOK Online [Database]. Retrieved September 2017. Available at: https://www.ibm.com/us-en/marketplace/micromedex-red-book. Accessed September 23, 2019.

28. Bristol-Myers Squibb. A reference guide to reimbursement and coding: Yerovy (ipilimumab). May 2019. Available at: http://www.bmsaccesssupport bmscustomerconnect.com/servlet/servlet.FileDownload?file=00Pi000000nz XevEAE. Accessed September 24, 2019.

29. Long GV, Flaherty KT, Stroyakovskiy D, et al. Dabrafenib plus trametinib versus dabrafenib monotherapy in patients with metastatic BRAF V600E/K-mutant melanoma: long-term survival and safety analysis of a phase 3 study. Ann Oncol. 2017;28(7):1631-39.

30. Opdivo (nivolumab) injection for intravenous use. Bristol-Myers Squibb September 2019. Available at: https://packageinserts.bms.com/pi/pi_opdivo. pdf. Accessed September 24, 2019.

31. Bly CA, Molife C, Brown J, et al. The budget impact of including necitumumab on the formulary for first-line treatment of metastatic squamous non-small cell lung cancer: U.S. commercial payer and Medicare perspectives. J Manag Care Spec Pharm. 2018;24(6):534-43. Available at: https:// www.jmcp.org/doi/10.18553/jmcp.2018.24.6.534.

32. Flannery K, Drea E, Hudspeth L, et al. Budget impact of cabazitaxel use after docetaxel treatment for metastatic castration-resistant prostate cancer. J Manag Care Spec Pharm. 2017;23(4):416-26. Available at: https://www.jmcp. org/doi/10.18553/jmcp.2017.23.4.416.

33. Bajaj PS, Veenstra DL, Goertz HP, Carlson JJ. Targeted erlotinib for first-line treatment of advanced non-small cell lung cancer: a budget impact analysis. J Med Econ. 2014;17(8):538-46

34. Xie J, Diener M, De G, Yang H, Wu EQ, Namjoshi M. Budget impact analysis of everolimus for the treatment of hormone receptor positive, human epidermal growth factor receptor-2 negative (HER2-) advanced breast cancer in the United States. J Med Econ. 2013;16(2):278-88.
35. Carlson JJ, Wong WB, Veenstra DL, Reyes C. Budget impact of erlotinib for maintenance therapy in advanced non-small cell lung cancer. J Med Econ. 2011;14(2):159-66.

36. Ho J, Zhang L, Todorova L, Whillans F, Corey-Lisle P, Yuan Y. Budget impact analysis of ixabepilone used according to FDA approved labeling in treatment-resistant metastatic breast cancer. J Manag Care Pharm. 2009;15(6):467-75. Available at: https://www.jmcp.org/doi/10.18553/ jmcp.2009.15.6.467.

37. Bloudek L, Roy A, Kish JK, et al. Estimating the economic impact of adding panobinostat to a U.S. formulary for relapsed and/or refractory multiple myeloma: a budget impact and cost-benefit model. J Manag Care Spec Pharm. 2016;22(8):991-1002. Available at: https://www.jmcp.org/doi/10.18553/ jmcp.2016.22.8.991

38. Bui CN, O'Day K, Flanders S, et al. Budget impact of enzalutamide for chemotherapy-naive metastatic castration-resistant prostate cancer. J Manag Care Spec Pharm. 2016;22(2):163-70. Available at: https://www.jmcp.org/ doi/10.18553/jmcp.2016.22.2.163.

39. Institute for Clinical and Economic Review. Overview of the ICER value assessment framework and update for 2017-2019. 2019. Available at: http://icer-review.org/wp-content/uploads/2017/06/ICER-value-assessmentframework-Updated-050818.pdf. Accessed September 28, 2019.

40. Stav I, Gyawali B, Goldstein DA. Duration of adjuvant immunotherapybiologic, clinical and economic considerations. Med Oncol. 2018;35(12):160

41. Francken AB, Accortt NA, Shaw HM, et al. Follow-up schedules after treatment for malignant melanoma. Br J Surg. 2008;95(11):1401-07.

42. Centers for Medicare \& Medicaid Services. CMS clinical diagnostic laboratory fee schedule [Database]. 2017. Available at: https://www.cms.gov/ apps/ama/license.asp?file=/Medicare/Medicare-Fee-for-Service-Payment/ ClinicalLabFeeSched/Downloads/17CLAB.zip. Accessed September 28, 2019

43. THxID-BRAF (package insert). BioMerieux. 2013. Available at: http:// www.biomerieux-usa.com/sites/subsidiary_us/files/braf-package-insert-1.pdf. Accessed September 28, 2019.

44. Centers for Medicare \& Medicaid Services. CMS physician fee schedule relative value file [Database]. 2017. Available at: https://www.cms. gov/Medicare/Medicare-Fee-for-Service-Payment/PhysicianFeeSched/ Downloads/RVU17A.zip. Accessed September 28, 2019.

45. MPI Group. The cost of dispensing study: an independent comparative analysis of U.S. prescription dispensing costs. September 2015. Available at: https://www.illinois.gov/hfs/SiteCollectionDocuments/ ReferencelTheCostofDispensingStudyCCPA.pdf. Accessed September 28, 2019.

46. Wang J, Chmielowski B, Pellissier J, Xu R, Stevinson K, Liu FX. Costeffectiveness of pembrolizumab versus ipilimumab in ipilimumab-naive patients with advanced melanoma in the United States. J Manag Care Spec Pharm. 2017;23(2):184-94. Available at: https://www.jmcp.org/doi/10.18553/ jmcp.2017.23.2.184

47. Eggermont AM, Chiarion-Sileni V, Grob JJ, et al. Adjuvant ipilimumab versus placebo after complete resection of high-risk stage III melanoma (EORTC 18071): a randomised, double-blind, phase 3 trial. Lancet Oncol. 2015;16(5):522-30.

48. McMasters KM, Egger ME, Edwards MJ, et al. Final results of the Sunbelt Melanoma Trial: a multi-institutional prospective randomized phase III study evaluating the role of adjuvant high-dose interferon alfa-2b and completion lymph node dissection for patients staged by sentinel lymph node biopsy. J Clin Oncol. 2016;34(10):1079-86

49. Arondekar B, Curkendall S, Monberg M, et al. Economic burden associated with adverse events in patients with metastatic melanoma. J Manag Care Spec Pharm. 2015;21(2):158-64. Available at: https://www.jmcp.org/ doi/10.18553/jmcp.2015.21.2.158. 
Budget Impact of Dabrafenib and Trametinib in Combination as Adjuvant Treatment of BRAF V600E/K Mutation-Positive Melanoma from a U.S. Commercial Payer Perspective

\begin{tabular}{|c|c|c|c|c|c|}
\hline APPENDIX A & \multicolumn{5}{|c|}{$\begin{array}{l}\text { Estimates of the Incidence of } \\
\text { Grades 3-5 Adverse Events }\end{array}$} \\
\hline Grade 3+ Adverse Event & $\begin{array}{c}\mathrm{D}+\mathrm{T} \\
(\mathrm{n}=435)\end{array}$ & $\begin{array}{c}\text { OBS } \\
(n=432)\end{array}$ & $\begin{array}{c}\text { HDI } \\
(\mathrm{n}=548)\end{array}$ & $\begin{array}{c}\text { IPI } \\
(\mathrm{n}=928)\end{array}$ & $\begin{array}{c}\text { NIVO } \\
(\mathrm{n}=453)\end{array}$ \\
\hline ALT increased, \% & 4 & 0 & 6 & 5 & 2 \\
\hline Constitutional/chills, \% & 1 & 0 & 13 & 0 & 0 \\
\hline Diarrhea, \% & 1 & 0 & 0 & 10 & 2 \\
\hline Granulocytopenia, \% & 0 & 0 & 17 & 0 & 0 \\
\hline Hypertension, \% & 6 & 2 & 0 & 0 & 0 \\
\hline Hypotension, \% & 0 & 0 & 0 & 0 & 0 \\
\hline Leukopenia, \% & 0 & 0 & 6 & 0 & 0 \\
\hline Liver toxicity, \% & 0 & 0 & 11 & 0 & 0 \\
\hline Malaise/fatigue, \% & 4 & 0 & 16 & 2 & 1 \\
\hline Myalgia, \% & 0 & 0 & 7 & 0 & 0 \\
\hline Nausea, \% & 1 & 0 & 5 & 0 & 0 \\
\hline Neuroclinical, \% & 0 & 0 & 8 & 0 & 0 \\
\hline Neurologic, \% & 0 & 0 & 7 & 0 & 0 \\
\hline Pyrexia, \% & 5 & 0 & 0 & 1 & 0 \\
\hline
\end{tabular}

$A L T=$ alanine aminotransferase; $D+T=$ dabrafenib plus trametinib combination; $H D I=$ high dose interferon-alpha $2 b ; I P I=$ ipilimumab; $N I V O=$ nivolumab;

OBS = observation

\begin{tabular}{l|c|c}
\hline \multicolumn{1}{c}{ APPENDIX B } & $\begin{array}{l}\text { Estimates of Direct Medical Costs } \\
\text { for Treatment of Grades 3-5 } \\
\text { Adverse Events }\end{array}$ \\
\hline Adverse Event & 2012 Cost per AE (\$) & 2017 Cost per AE (\$) \\
\hline ALT increased & $4,764.95$ & $5,582.50$ \\
\hline Constitutional/chills & $8,243.64$ & $9,658.06$ \\
\hline Diarrhea & $4,443.15$ & $5,205.49$ \\
\hline Granulocytopenia & $8,502.98$ & $9,961.89$ \\
\hline Hypertension & $9,629.61$ & $11,281.82$ \\
\hline Leukopenia & $8,502.98$ & $9,961.89$ \\
\hline Liver toxicity & $14,745.51$ & $17,275.50$ \\
\hline Malaise/fatigue & $4,534.97$ & $5,313.06$ \\
\hline Myalgia & $4,764.95$ & $5,582.50$ \\
\hline Nausea & $5,760.56$ & $6,748.93$ \\
\hline Neuroclinical & $5,902.59$ & $6,915.33$ \\
\hline Neurologic & $5,902.59$ & $6,915.33$ \\
\hline Pyrexia & $8,243.64$ & $9,658.06$ \\
\hline AE adverse event; ALT=alanine aminotransferase. \\
\hline
\end{tabular}

